University of Texas at El Paso

ScholarWorks@UTEP

4-2005

\title{
From Fuzzification and Intervalization to Anglification: A New 5D Geometric Formalism for Physics and Data Processing
}

\author{
Scott A. Starks \\ The University of Texas at El Paso, sstarks@utep.edu \\ Vladik Kreinovich \\ The University of Texas at El Paso, vladik@utep.edu
}

Follow this and additional works at: https://scholarworks.utep.edu/cs_techrep

Part of the Computer Engineering Commons

Comments:

UTEP-CS-05-16.

Published in Proceedings of the 24th International Conference of the North American Fuzzy Information Processing Society NAFIPS'2005, Ann Arbor, Michigan, June 22-25, 2005, pp. 401-406.

\section{Recommended Citation}

Starks, Scott A. and Kreinovich, Vladik, "From Fuzzification and Intervalization to Anglification: A New 5D Geometric Formalism for Physics and Data Processing" (2005). Departmental Technical Reports (CS). 245.

https://scholarworks.utep.edu/cs_techrep/245

This Article is brought to you for free and open access by the Computer Science at ScholarWorks@UTEP. It has been accepted for inclusion in Departmental Technical Reports (CS) by an authorized administrator of ScholarWorks@UTEP. For more information, please contact Iweber@utep.edu. 


\title{
From Fuzzification and Intervalization to Anglification: A New 5D Geometric Formalism for Physics and Data Processing
}

\author{
Scott A. Starks and Vladik Kreinovich \\ NASA Pan-American Center for Earth and Environmental Studies (PACES) \\ University of Texas at El Paso, El Paso, TX 79968, USA \\ \{sstarks,vladik\}@utep.edu
}

\begin{abstract}
We show that in understanding foundations of modern physics, with its 10-dimensional (and higher-dimensional) space-time models, it is very helpful to use the main ideas behind fuzzification - extension of arithmetic operations and elementary functions from exact numbers to fuzzy numbers. The resulting formalism is, from the mathematical viewpoint, somewhat more complex than the traditional fuzzy arithmetic, but it is still much simpler than the quantum field theory - and thus, it helps to make several important ideas from foundations of modern physics much more intuitively clear.
\end{abstract}

\section{Data Processing: GeOMETRIC InTERPRETATION IS} NEEDED

Our interest originated from the needs of data processing; the data to be processed consists of values of several measured quantities, i.e., usually, several real numbers. In the computer, the sequence of real numbers $\left(x_{1}, \ldots, x_{n}\right)$ is represented as an array, i.e., in mathematical terms, as an $n$-dimensional vector (an element of an $n$-dimensional space).

This reformulation in terms of $n$-dimensional geometry often enables us to reformulate non-visual notions from data processing in more visual geometric terms. For example:

- When we use Gaussian distribution, then the level sets of this distribution forms ellipsoids in this n-dimensional space.

- An a priori relation between the variables, if linear, describes a plane in this n-dimensional space.

For small $n$ ( $\leq 3$ or 4 ), this geometric interpretation is often helpful for data processing, because we can then use: our geometric intuition and geometric results developed in geometry and physics to simplify data processing methods.

It would be nice to have similar geometric techniques for larger $n$, but for larger $n$, we do not have ready-made geometric results to apply:

- our geometric intuition does not work that well in multidimensional spaces, and

- physical results are no longer applicable, because physics is mainly interested in 3D space and 4D space-time.

To get such results, we turned to the areas of physics where multi-dimensional geometries are currently used.

\section{Physics: 5D GeOMetry is USEFUL}

After the 1916 success of A. Einstein, who explained gravitation by combining space and time into a 4D space, there have been many efforts to explain other physical fields by adding other physical dimensions.

The first successful attempt was made by Th. Kaluza and O. Klein in 1921. They showed that if we formally consider the equations of general relativity theory in the $5 \mathrm{D}$ space, the equations for the normal $4 \times 4$ components $g_{i j}$ of the metric tensor still describe gravitation, while the new components $g^{5 i}$ of the metric tensor satisfy Maxwell's equations (under the assumption that $g_{55}=$ const). Thus, if we go to 5D space, we get a geometric interpretation of electrodynamics.

The only problem with this interpretation is that it is formal: change in first 4 dimensions makes perfect physical sense, while there seemed to be no physical effects corresponding to change in 5th dimension. To solve this problem, A. Einstein and P. Bergmann proposed, in 1938 [1], that the 5th dimension forms a tiny circle, so that only micro-particles "see" it, while for us, the world is 4D.

This is a standard view now in particle physics; see, e.g., [2], [10]: space is 10- or 11-dimensional, all dimensions except the first four are tiny.

\section{The Physical Model is Unusual, But This} UN-USUALNESS IS APPROPRIATE FOR DATA PROCESSING

The standard multi-D physical model is unusual geometrically: the space is a cylinder, not a plane anymore. This feature is, however, interestingly related to data processing: some measured data are angles, and angles do form a circle, so these geometric ideas can be directly applied to data processing.

\section{FORMULAS FROM PHYSICAL 5D THEORIES THAT} NeEd to Be Explained in Purely Geometric Terms

In addition to a nice geometric model, the traditional description of Kaluza-Klein theory requires several additional physical formulas, formulas that look very artificial because they do not have a direct geometric explanation.

In this paper, we will show that, if we take the EinsteinBergmann model seriously, then these formulas can be derived - and thus, they are not additional and ad hoc.

What are these formulas that do not directly follow from the geometric model?

First, the assumption $g_{55}=$ const is artificial. 
Second, since only four coordinates have a physical sense, the distance $\Delta s^{2}=\sum_{i=1}^{5} \sum_{j=1}^{5} g_{i j} \cdot \Delta x_{i} \cdot \Delta x_{j}$ between the points $x$ and $x+\Delta x$ should only depend on the first 4 coordinates - while in general, for a 5D metric, the terms $g_{55} \cdot\left(\Delta x^{5}\right)^{2}$ and $g_{5 i} \cdot \Delta x^{5} \cdot \Delta x^{i}$ create a difficult-to-explain dependence on $\Delta x^{5}$.

Third, we would like to explain the fact that the observed values of physical fields do not depend on the fifth coordinate $x^{5}$, e.g., that $\partial g_{i j} / \partial x^{5}=0$ (this condition is called cylindricity).

Several other formulas came from the attempts to give the fifth dimension a physical interpretation. Namely, in the 1940s, Yu. Rumer showed (see, e.g., [11]) that if we interpret $x^{5}$ as action $S=\int L d x d t$ (i.e., the quantity whose extrema define the field's dynamics), then the fact that $x^{5}$ is defined on a circle is consistent with the fact that in quantum physics (e.g., in its Feynman integral formulation), action is used only as part of the expression $\exp (i S / h)$, whose value is not changed if we add a constant $2 \pi \cdot h$ to $S$. (For a $\mathrm{H}$ atom, this idea leads to the original Bohr's quantization rules.)

Action is defined modulo arbitrary transformation $S \rightarrow S+$ $f\left(x^{i}\right)$; thus, the corresponding transformation $x^{5} \rightarrow x^{5}+f\left(x^{i}\right)$ should be geometrically meaningful. Similar transformations stem from the electrodynamic interpretation of $g_{5 i}$ as $A_{i}$ : gauge transformations $A_{i} \rightarrow A_{i}-\partial f / \partial x_{i}$.

\section{NATURAL IDEA AND ITS PROBlems}

The main difference between a standard 4D space and Einstein-Bergmann's 5D model is that we have a cylinder $K=R^{4} \times S^{1}$ ( $K$ for Kaluza) instead of a linear space. It is, therefore, desirable to modify standard geometry by substituting $K$ instead of $R^{4}$ into all definitions.

The problem with this idea is that the corresponding formalisms of differential geometry use the underlying linear space structure, i.e., addition and multiplication by a scalar. We still have addition in $K$, but multiplication is not uniquely defined for angle-valued variables: we can always interpret an angle as a real number modulo the circumference, but then, e.g., $0 \sim 2 \pi$ while $0.6 \cdot 0 \nsim 0.6 \cdot 2 \pi$.

\section{What We Suggest}

We do need a real-number representation of an angle variable. A more natural representation of this variable is not as a single real number, but as a set $\{\alpha+n \cdot 2 \pi\}$ of all possible real numbers that correspond to the given angle.

Similarly to interval and fuzzy arithmetic, we can naturally define element-wise arithmetic operations on such sets, e.g., $A+B=\{a+b \mid a \in A, b \in B\}$. We can then define tensors as linear mappings that preserve the structure of such sets, and we can define a differentiable tensor field as a field for which the set of all possible values of the corresponding partial derivatives is also consistent with the basic structure.

Comment. These results were first announced in [6], [7], [12].

\section{RESULTING FORMALISM: IDEA}

In mathematical terms, the resulting formalism is equivalent to the following: We start with the space $K$ which is not a vector space (only an Abelian group). We reformulate standard definitions of vector and tensor algebra and tensor analysis and apply them to $K: K$-vectors are defined as elements of $K ; K$ covectors as elements of the dual group, etc. All physically motivated conditions turn out to be natural consequences of this formalism.

\section{VIII. $K$-VECTORS}

In the traditional 4-D space-time $R^{4}$, we can define a vector as simply an element of $R^{4}$. In our case, instead of 4-D spacetime $R^{4}$, we have a 5-D space-time $K \stackrel{\text { def }}{=} R^{4} \times S^{1}$, in which $S^{1}$ is a circle of a small circumference $h>0$ - i.e., equivalently, a real line in which two numbers differing by a multiple of $h$ describe the same point: $\left(x^{1}, \ldots, x^{4}, x^{5}\right) \sim\left(x^{1}, \ldots, x^{4}, x^{5}+\right.$ $k \cdot h)$. Thus, it is natural to define $K$-vectors as simply elements of $K$ :

Definition 1. A $K$-vector is an element of $K=R^{4} \times S^{1}$.

On the set of all vectors in $R^{4}$, there are two natural operations: (commutative) addition $a+b$ and multiplication by a real number $\lambda: a \rightarrow \lambda \cdot a$. Thus, this set is a linear space.

In contrast, on the the set $K$ of all $K$-vectors we only have addition, so the set of all $K$-vectors is not a linear space, it is only an Abelian group.

\section{IX. $K$-COVECTORS}

In physics, an important algebraic object is a covector: vectors describe the location $x$ of a particle, while the corresponding covector $p$ describes the energy and momentum of the corresponding particle. Because of this physical importance, it is necessary to generalize the notion of covectors to the new space.

We would like to provide a generalization that preserves the physical meaning of the connection between vectors and covectors. The physical connection is probably best described in quantum mechanics. In quantum mechanics, due to Heisenberg's uncertainty principle $\Delta x \cdot \Delta p \geq \hbar$, if we know the exact location of a particle (i.e., if $\Delta x=0$ ), then we have no information about the momentum (i.e., $\Delta p=\infty$ ), and vice versa, if we know the exact momentum $(\Delta p=0)$, then we have no information about the particle's location. In other words, if we have a state with a definite momentum $p$, and we then shift the coordinates by a vector $t$, i.e., replace $x$ by $x+t$, the known state of the particle should not change.

In quantum mechanics, a state of the particle is described by a complex-valued function $\psi(x)$ called a wave function. The wave function itself is not directly observable, what we observe are probabilities $|\psi|^{2}$. So, if we multiply all the values of the wave-function by a complex number $\varphi$ with $|\varphi|=1$ (i.e., by a number of the type $\exp (\mathrm{i} \cdot \alpha)$, where $\mathrm{i}=\sqrt{-1}$ and $\alpha$ is a real number), then all the probabilities remain the same - i.e., from the physical viewpoint, we will have exactly the same state. Thus, for every real number $\alpha$, the functions 
$\psi(x)$ and $\exp (\mathrm{i} \cdot \alpha) \cdot \psi(x)$ describe exactly the same state. When we say that the state $\psi(x)$ does not change after shift $x \rightarrow x+t$, we mean that the original function $\psi(x)$ and the function $\psi(x+t)$ that describe the shifted state describe the same state - i.e., $\psi(x+t)=\varphi(t) \cdot \psi(x)=\exp (\mathrm{i} \cdot \alpha(t)) \cdot \psi(x)$ for some complex number $\varphi(t)$ or, equivalently, real number $\alpha(t)$ (which, generally speaking, depends on the shift $t$ ).

Since $\exp (\mathrm{i} \cdot 2 \cdot \pi)=1$, the value $\alpha(t)$ is only determined modulo $2 \cdot \pi$. Thus, $\alpha(t)$ is a point on a circle rather than a real number.

For $x=0$, we get $\psi(t)=\varphi(t) \cdot \psi(0)$, so modulo a multiplicative constant, shift-invariant states $\psi(t)$ are equal to the corresponding functions $\varphi(t)$. So, to determine such states, we must describe all the corresponding functions $\varphi(t)$.

When we shift by $t=0$, the function remains unchanged, i.e., $\varphi(0)=1$ (equivalently, $\alpha(0)=0$ ).

If we first shift $t$ and then by $s$, then we get the same result as if we shift once by $t+s$. Hence, we have

$$
\varphi(s) \cdot(\varphi(t) \cdot \psi(x))=\varphi(t+s) \cdot \psi(x),
$$

so $\varphi(t+s)=\varphi(t) \cdot \varphi(s)$. So, from the physical viewpoint, a shift-invariant state $\varphi$ is a mapping from $R^{4}$ to the unit circle $S^{1}=\{\varphi:|\varphi|=1\}$ that transform 0 into 1 and sum into sum. In mathematics, such a mapping is called a homomorphism from an Abelian additive group $R^{4}$ to $S^{1}$.

It is also physically reasonable to assume that the wave function is continuous - hence, that the homomorphism $\varphi$ is continuous. Continuous homomorphisms from an Abelian group $G$ to a unit circle are called characters; the set of all such characters is also an Abelian group called dual (and denoted by $\left.G^{*}\right)$. So, it is natural to associate covectors with elements of the dual group.

For $R^{4}$, this definition fits well with the more traditional one, because it is known that for $R^{4}$, the dual group is also $R^{4}$ : every character has the form $\exp (\mathrm{i} \cdot p \cdot x)$. For $K=R^{4} \times S^{1}$, we get a new definition:

Definition 2. A $K$-covector is a character of the group $K$, i.e., a continuous homomorphism from $K$ to $S^{1}$. By a sum of two covectors we mean the product of the corresponding homomorphisms.

The set of all $K$-covectors is thus a dual group $K^{*}$ to $K$. It is known that elements of this dual group have the form $\exp (\mathrm{i} \cdot p \cdot x)$, where $p=\left(p_{1}, \ldots, p_{4}, p_{5}\right), p_{1}, \ldots, p_{4}$ can be any real numbers, and $p_{5}$ is an multiple of $1 / h$. Thus, the group $K^{*}$ of all $K$-covectors is isomorphic to $R^{4} \times Z$, where $Z$ is the additive group of all integers.

Comment. $K$-vectors are simply elements $x=\left(x_{1}, \ldots, x_{5}\right)$ of $R^{5}$, some of which are equivalent to each other: $x \sim x^{\prime}$ if $x_{5}-x_{5}^{\prime}=k \cdot h$ for some integer $k$. In other words, a $K$-vector can be viewed as a set

$$
\left\{x^{\prime}: x^{\prime} \sim x\right\}=\left\{\left(x_{1}, \ldots, x_{4}, x_{5}+k \cdot h\right\} .\right.
$$

A unit circle $S^{1}$ can also be described as simply the set $R$ of all real numbers with the equivalence relation $\alpha \sim \alpha^{\prime}$ if and only if $\alpha-\alpha^{\prime}=k \cdot(2 \cdot \pi)$ - or, equivalently, as the class of sets $\{\alpha+k \cdot(2 \cdot \pi)\}$.

In these terms, we can alternative describe $K$-covectors as linear mappings $x=\left(x_{1}, \ldots, x_{5}\right) \rightarrow p \cdot x=\sum p_{i} \cdot x_{i}$ from $R^{5}$ to $R$ that are consistent with the above structures, i.e., mapping for which $x \sim x^{\prime}$ implies $p \cdot x \sim p \cdot x^{\prime}$.

\section{X. $K$-Tensors}

To describe individual particles, it is usually sufficient to consider vectors (that describe their location) and covectors (that describe their momentum). However, to describe field theories such as Maxwell's theory of electromagnetism or Einstein's General Relativity theory, it is not sufficient to consider only vectors and covectors, we also need to consider tensors.

Specifically, for $G=R^{4}$, for every two integers $p \geq 0$ and $q \geq 0$, a tensor of valence $(p, q)$ can be defined as a multilinear map $G^{p} \times\left(G^{*}\right)^{q} \rightarrow R$ - where multi-linear means that if we fix the values of all the variables but one, we get a linear mapping. Every such multi-linear mapping has the form

$$
\begin{gathered}
x^{i_{1}}, \ldots, y^{i_{p}}, z_{j_{1}}, \ldots, u_{j_{q}} \rightarrow \\
\sum_{i_{1}, \ldots, i_{p}, j_{1}, \ldots, j_{q}} t_{i_{1} \ldots i_{p}}^{j_{1} \ldots j_{q}} \cdot x^{i_{1}} \cdot \ldots \cdot y^{i_{p}} \cdot z_{j_{1}} \cdot \ldots \cdot u_{j_{q}}
\end{gathered}
$$

for some components $t_{i_{1} \ldots i_{p}}^{j_{1} \ldots j_{q}}$. We thus naturally arrive at the following definition:

Definition 3. Let $G_{1}, \ldots, G_{m}, G$ be continuous Abelian groups. A mapping $t: G_{1} \times \ldots \times G_{m} \rightarrow G$ is called $Z$ multilinear if for every $i$, if we fix the values of all the variables except $i$-th, we get a homomorphism.

Definition 4. Let $p \geq 0$ and $q \geq 0$. By a $K$-tensor of valence $(p, q)$, we mean a continuous $Z$-multilinear mapping $t: K^{p} \times$ $\left(K^{*}\right)^{q} \rightarrow S^{1}$.

Comments. For $R^{4}$ instead of $K$, this definition coincides with the traditional one.

When $K=R^{4} \times S^{1}$, this definition is consistent with the previous ones: $K$-tensors of valence $(0,1)$ are $K$-covectors, and $K$-tensors of valence $(1,0)$ are $K$-vectors.

This definition can be reformulated as follows: a $K$-tensor is a multi-linear mapping that is consistent with the equivalence sets structure, i.e., for which $x \sim x^{\prime}, \ldots, y \sim y^{\prime}$ implies that $t(x, \ldots, y, z, \ldots, u) \sim t\left(x^{\prime}, \ldots, y^{\prime}, z, \ldots, u\right)$.

Two multi-linear mappings $t$ and $t^{\prime}$ describe the same $K$ tensor if $t(x, \ldots, y, z, \ldots, u) \sim t^{\prime}(x, \ldots, y, z, \ldots, u)$ for all $x, \ldots, y, z, \ldots, u$.

The following result describes all such mappings:

\section{Proposition 1.}

- Every K-tensor has the form

$\exp \left(\mathrm{i} \cdot \sum_{i_{1}, \ldots, i_{p}, j_{1}, \ldots, j_{q}} t_{i_{1} \ldots i_{p}}^{j_{1} \ldots j_{q}} \cdot x^{i_{1}} \cdot \ldots \cdot y^{i_{p}} \cdot z_{j_{1}} \cdot \ldots \cdot u_{j_{q}}\right)$ 
for some components $t \ldots$. In this representation, of all the components in which one of the lower indices is 5, only a component $t_{5}^{5 \ldots 5}$ can be non-zero, and it can only take values $2 \cdot \pi \cdot h^{q-1} \cdot k$ for some integer $k$.

- Vice versa, if we have a set of components $t_{\ldots}^{\ldots}$ in which of all the components in which one of the lower indices is 5, only a component $t_{5}^{5 \ldots 5}$ may be non-zero, its value is $2 \cdot \pi \cdot h^{q-1} \cdot k$ for some integer $k$, then the above formula defines a $K$-tensor.

- Two sets of components $t^{\cdots}$ and $s^{\cdots}$.. define the same $K$ tensor if and only if all their components coincides with a possible exception of components $t^{5 \ldots 5}$ and $s^{5 \ldots 5}$ which may differ by $2 \cdot \pi \cdot h^{q} \cdot k$ for an integer $k$.

Comment. For readers' convenience, all the proofs are given in the Appendix.

\section{EXPLAINING THE CONDITION $g_{55}=$ const AND THE FACT THAT Metric Does Not DEPEND ON $x^{5}$}

For $g_{i j}$, Proposition 1 implies that $g_{55}=g_{5 i}=0$. Thus, the above geometric formalism explains the first two physical assumptions that we wanted to explain: that $g_{55}=0$ and that the distance $\Delta s^{2}=\sum_{i=1}^{5} \sum_{j=1}^{5} g_{i j} \cdot \Delta x_{i} \cdot \Delta x_{j}$ between the two points $x$ and $x+\Delta x$ only depends on their first 4 coordinates.

\section{Differential Formalism FOR $K$-TEnSOR FIELdS}

Definition 5. By a $K$-tensor field $f_{\ldots} \ldots(x)$ of valence $(p, q)$, we mean a mapping that assigns, to every point $x \in K, a$ $K$-tensor $f_{\ldots} \ldots(x)$ of this valence.

Most physics is described in the language of differential equations. It is known that for every tensor field $t_{i_{1} \ldots i_{p}}^{j_{1} \ldots j_{q}}$ of valence $(p, q)$, its gradient $\partial t_{i_{1} \ldots i_{p}}^{j_{1} \ldots j_{q}} / \partial x^{m}$ is also a tensor field - of valence $(p+1, q)$. This new field is called a gradient tensor field. It is therefore natural to give the following definition:

Definition 6. We say that a $K$-tensor field of valence $(p, q)$ is differentiable if the corresponding component tensor field is continuously differentiable, and its gradient field also defines a K-tensor field.

In other words, to differentiate a $K$-tensor field, we form the corresponding tensor field, differentiate it, and then interpret the result as a $K$-tensor field of valence $(p+1, q)$. When is this possible? The answer to this question is as follows:

Proposition 2. The $K$-tensor field is differentiable if and only if all its components $t_{\ldots}^{\ldots}$ do not depend on $x^{5}$, with the possible exception of the component $t^{5 \ldots 5}$ which may have the form $2 \cdot \pi \cdot h^{q-1} \cdot x^{5}+f\left(x_{1}, \ldots, x_{4}\right)$.

\section{CYLINDRICITY EXPLAINED}

As a result of Proposition 2, we conclude that for all the components $t$ (except for angular-valued ones), we have the cylindricity condition $\partial t_{\ldots}^{\cdots} / \partial x^{5}=0$. Thus, the cylindricity conditions is also explained by the geometric model.

\section{Linear CoORdinate Transformations}

In the traditional affine geometry, in addition to shifts, we can also consider arbitrary linear coordinates transformations. In geometric terms, we can define these transformations as continuous automorphisms of the additive group $K_{0}=R^{4}$. We can define vectors and tensors as continuous homomorphisms $T: K_{0}^{p} \times\left(K_{0}^{*}\right)^{q} \rightarrow S^{1}$; in this case, e.g., standard formulas for transforming covectors (i.e., continuous homomorphisms $g: K_{0} \rightarrow S^{1}$ ) can be uniquely determined by the requirement that the value $g(a)$ be preserved under such a transformation, i.e., that $g^{\prime}\left(a^{\prime}\right)=g(a)$. Similarly, the transformation law for tensors can be determined by the condition that

$$
t^{\prime}\left(a_{1}^{\prime}, \ldots, a_{p}^{\prime}, b_{1}^{\prime}, \ldots, b_{q}^{\prime}\right)=t\left(a_{1}, \ldots, a_{p}, b_{1}, \ldots, b_{1}\right) .
$$

Similarly, for $K=R^{4} \times S^{1}$, we can define a $K$-linear transformation as follows:

Definition 7. By a $K$-linear transformation, we mean a continuous automorphism of the additive group of $K$.

Proposition 3. Every $K$-linear transformation has the form

$$
x^{5} \rightarrow \pm x^{5}+\sum_{i=1}^{4} A_{i} \cdot x^{i} ; \quad x^{i} \rightarrow \sum_{j=1}^{4} b_{j}^{i} x^{j}, \quad(i \leq 4) .
$$

The corresponding tensor transformations can be defined by the condition (1). Once can see that in this case, the tensor components are transformed just like the normal tensor components. In particular, under the above $K$-linear transformation, a covector is transformed as follows:

$$
x_{5} \rightarrow \pm x_{5}, \quad x_{i} \rightarrow \sum_{i=1}^{4} c_{i}^{j} x_{j}-A_{i} \cdot x_{5},
$$

where $c_{i}^{j}$ is the matrix that is inverse to $b_{j}^{i}$.

\section{General Coordinate Transformations}

Definition 8. A smooth transformation $s: K \rightarrow K$ is admissible if and only if for each point $x \in K$, the corresponding tangent transformation

$$
a^{i} \rightarrow a_{\text {new }}^{i}=\sum_{j=1}^{5} \frac{\partial s^{i}}{\partial x^{j}} a_{\mid x}^{i}
$$

is a K-linear transformation.

Proposition 4. Every admissible transformation has the form

$$
x^{5} \rightarrow \pm x^{5}+f\left(x^{1}, \ldots, x^{4}\right), \quad x^{i} \rightarrow f^{i}\left(x^{1}, \ldots, x^{4}\right) .
$$

Comment. We have already mentioned that functions on $K=$ $R^{4} \times S^{1}$ are simply functions on $R^{5}$ which are periodic in $x^{5}$ with the period $h$. Also, a $K$-covector $p$ can be simply viewed as a covector for which the fifth component $p_{5}$ is an integer multiple of $1 / h$. Thus,, e.g., a $K$-covector field on $K$ can be viewed as a covector field $p(x)=\left(p_{1}(x), \ldots, p_{5}(x)\right)$ on $R^{5}$ that satisfies the following two properties: 
(a) this field is periodic in $x^{5}$ with period $p$;

(b) for each $x$, the value $p_{5}(x)$ is an integer multiple of $1 / h$.

It is therefore reasonable to define a general coordinate transformation of $K$ as a coordinate transformation of $R^{5}$ that preserves this property, i.e., under which a covector field that satisfies the properties (a) and (b) are transformed into a covector field that also satisfies these properties. One can see that this leads to the same class of general coordinate transformations.

\section{Gauge Transformations Explained}

According to Proposition 4, every admissible transformation is a composition of a $4 \mathrm{D}$ transformation and an additional gauge transformation $x^{5} \rightarrow x^{5}+f\left(x^{1}, \ldots, x^{4}\right)$ - exactly as described by Rumer.

\section{Case of Curved Space-Time}

In modern physics, space-time is a manifold, i.e., a topological space $V$ which is locally diffeomorphic to $R^{4}$. Since our basic model is not $R^{4}$, but $K=R^{4} \times S^{1}$, it is reasonable to define a $K$-manifold as a topological space that is locally diffeomorphic to $K$.

From the mathematical viewpoint, $K$ is $R^{5}$ factorized over the vector $e=(0, \ldots, 0, h)$ : i.e., $a \sim b$ if and only if $a-b$ is an integer multiple of $e$. Thus, a natural way to describe a $K$-manifold is to describe a standard 5D manifold in which we have a vector $e(x)$ in every tangent space - i.e., a manifold with an additional vector field.

In this case, every tangent space is isomorphic to $K$. Thus, a $K$-tensor field can be defined as a mapping that maps every point $x \in V$ into a $K$-tensor defined over the space $K$ which is tangent at $x$.

\section{Auxiliary Result: Why There Is No} Physically Useful Gravitational Analog of Hertz POTENTIAL

In electromagnetism, in addition to the electromagnetic file $F_{i j}$ and the potential $A_{i}$ from which this filed can be obtained by differentiation $F_{i j}=\partial A_{i} / \partial x_{j}-\partial A_{j} / \partial x_{i}$, there is also a useful notion of a Hertz potential $H^{i k}$ for which $A^{i}$ can be obtained by differentiation $A^{i}=\sum_{k} \partial H^{i k} / \partial x^{k}$.

In gravitation, the natural analogy of potentials $A_{i}$ is the gravity tensor filed $g^{i j}$. From the purely mathematical viewpoint, it is possible to introduce a gravitational analog of the Hertz potential: namely, there exists a tensor field $\Pi^{i j k}$ for which

$$
g^{i j}=\sum_{k} \frac{\partial \Pi^{i j k}}{\partial x^{k}}
$$

see, e.g., [9]. However, in contrast to the electromagnetic case, this new potential does not seem to have any physical applications. Why?

Our explanation is simple: while (2) is impossible in the $4 \mathrm{D}$ case, it is no longer possible if we consider 5D $K$-tensor fields.
XiX. Potential Applications to Data Processing

For example, a natural analog of Gaussian distribution is $\exp \left(-\sum a_{i j} x^{i} x^{j}\right)$ for a $K$-tensor $a_{i j}$.

\section{ACKNOWLEDGMENTS}

The research was partially supported by NASA under cooperative agreement NCC5-209, by NSF grants EAR0112968, EAR-0225670, and EIA-0321328, and by NIH grant 3T34GM008048-20S1.

The authors are thankful to Professor L. Zadeh for describing a general scheme behind fuzzification and intervalization, a scheme that led us to the idea of anglification, and to all the participants of the special section of the October 1997 Montreal meeting of the American Mathematical Society, where physico-geometric aspects of this research were presented, for valuable comments; we are especially thankful to Prof. Abraham Ungar who organized this session, and to Yakov Eliashberg (Stanford) for important comments.

\section{REFERENCES}

[1] A. Einstein and P. Bergmann, "On the generalization of Kaluza's theory of electricity", Ann. Phys., 1938, Vol. 39, pp. 683-701.

[2] M. B. Green, J. H. Schwarz, and E. Witten, Superstring Theory, Vols. 1, 2, Cambridge University Press, 1988.

[3] Th. Kaluza, Sitzungsberichte der K. Prussischen Akademie der Wiseenschaften zu Berlin, 1921, p. 966 (in German); Engl. translation "On the unification problem in physics" in [8], pp. 1-9.

[4] O. Klein, Zeitschrift für Physik, 1926, Vol. 37, p. 895 (in German); Engl. translation "Quantum theory and five-dimensional relativity" in [8], pp. 10-23.

[5] O. M. Kosheleva and V. Kreinovich, A 5-dimensional geometric formalism for describing physical theories, Leningrad Center for New Information Technology "Informatika", Technical Report, Leningrad, 1989 (in Russian).

[6] V. Kreinovich and H. T. Nguyen, "Differentiation Beyond Traditional Definitions: Case Studies of Application-Motivated Extensions", $A b$ stracts of the 2005 Meeting of the Southwestern Section of the Mathematical Association of America (MAA), April 1-2, 2005, p. 6.

[7] V. Kreinovich and S. A. Starks, "A new 5D geometric formalism for physics and for data processing", Abstracts of American Mathematical Society, 1997, Vol. 18, No. 3, p. 460.

[8] H. C. Lee (ed.), An introduction to Kaluza-Klein theories, World Scientific, Singapore, 1984.

[9] M. Ya. Pal'chik, Institute of Nuclear Physics, Novosibirsk, Russia, Technical Report IYaF-325, 1969.

[10] J. Polchinski, String Theory, Vols. 1, 2, Cambridge University Press, 1998.

[11] Yu. B. Rumer, Studies in 5-optics, Gostekhizdat, Moscow, 1956.

[12] S. A. Starks and V. Kreinovich, "Non-Interval Extension of Interval Methods Leads to a New 5D Geometric Formalism for Physics and Data Processing", Abstracts of the International Conference on Interval Methods and their Application in Global Optimization (INTERVAL'98), April 20-23, 1998, Nanjing, China, pp. 136-138.

\section{APPENDIX: PROOFS}

\section{A. Proof of Proposition 1}

Let us first prove that every $K$-tensor can be described by the desired formula.

Indeed, let $t$ be a $K$-tensor. Let us first consider the restriction of $t$ to $K^{p} \times\left(R^{4}\right)^{q}$. Since locally, $K$ coincides with $R^{5}$, this restriction is, locally, a multi-linear map from $\left(R^{5}\right)^{p} \times\left(R^{4}\right)^{q}$ to $S^{1}$. Since it is multi-linear, at 0 , the value of this map is 1 . In a small vicinity of 1 , we can define a 
unique angle $(1 / \mathrm{i}) \cdot \ln t$. The resulting mapping is - locally a multi-linear mapping, in the traditional sense of this term, from $\left(R^{5}\right)^{p} \times\left(R^{4}\right)^{q}$ to $R$. Hence, in this vicinity, $\ln t=$ $\mathrm{i} \cdot \sum t_{\ldots} \cdot x^{i_{1}} \cdot \ldots$ So, for the restriction of $t$ to $K^{p} \times\left(R^{4}\right)^{q}$, we get the desired formula.

Similarly, for $K_{0} \stackrel{\text { def }}{=}\left(R^{5}\right)^{m} \times R^{4} \times \ldots \times R^{4} \ldots \times e \times R^{4} \times$ $\ldots \times R^{4}$, with $r$-th term replaced by $e \stackrel{\text { def }}{=}\left(0,0,0,0, h^{-1}\right)$, we conclude that the restriction of $t$ to $K_{0}$ has the form

$$
\exp \left(\mathrm{i} \cdot \sum t_{i_{1} \ldots i_{p}}^{j_{1} \ldots j_{r-1} 5 j_{r+1} \ldots j_{q}} \cdot x^{i_{1}} \cdot \ldots \cdot y^{i_{p}} \cdot z_{j_{1}} \cdot \ldots \cdot u_{j_{q}}\right)
$$

for some values $t_{i_{1} \ldots i_{p}}^{j_{1} \ldots j_{r-1} 5 j_{r+1} \ldots j_{q}}$. Since the restriction of $t$ to the $r$-th copy of $K^{*}$ is a homomorphism, this formula also holds for elements of $\left(R^{5}\right)^{m} \times R^{4} \times \ldots \times R^{4} \ldots \times Z \times R^{4} \times$ $\ldots \times R^{4}$,

Similar formulas hold for the subsets that can be obtained by replacing some of $K^{*}=R^{4} \times Z$ with $R^{4}$ and some by $Z$. Since $t$ is a homomorphism w.r.t. each of its variables, we can represent each element $p=\left(p_{1}, \ldots, p_{4}, p_{5}\right) \in K^{*}$ as a sum of $p^{(4)}=\left(p_{1}, \ldots, p_{4}, 0\right) \in R^{4}$ and $p^{(5)}=\left(0, \ldots, 0, p_{5}\right) \in Z$. For each of these two vectors, we have the desired formula; multiplying them, we get a similar formula for $p$. By using a similar decomposition w.r.t. other variables, we get the desired formula for all possible inputs from $K^{p} \times\left(K^{*}\right)^{q}$.

Let us now prove the desired properties of the components $t \ldots$. Since $t$ is defined on $K^{p} \times\left(K^{*}\right)^{q}$, replacing $x^{5}$ with $x^{5}+h$ should change the sum

$$
\sum_{i_{1}, i_{2}, \ldots, i_{p}, j_{1}, \ldots, j_{q}} t_{i_{1} i_{2} \ldots i_{p}}^{j_{1} \ldots j_{q}} \cdot x^{i_{1}} \cdot d^{i_{2}} \ldots \cdot y^{i_{p}} \cdot z_{j_{1}} \cdot \ldots \cdot u_{j_{q}}
$$

by an integer multiple of $2 \cdot \pi$. In other words, the difference between the new sum and old sum, i.e.,

$$
h \cdot \sum_{5, i_{2}, \ldots, i_{p}, j_{1}, \ldots, j_{q}} t_{5 i_{2} \ldots i_{p}}^{j_{1} \ldots j_{q}} \cdot d^{i_{2}} \cdot \ldots \cdot y^{i_{p}} \cdot z_{j_{1}} \cdot \ldots \cdot u_{j_{q}}
$$

must be a multiple of $2 \cdot \pi$ for all $d^{i_{2}}, \ldots, y^{i_{p}}$.

Let us first consider the case $p>1$. For $d^{i_{2}}=\ldots=y^{i_{p}}=$ 0 , the difference is equal to 0 ; this difference continuously depends on $d^{i_{2}}, \ldots, y^{i_{p}}$, and it is only allowed a discrete set of values. Due to continuity, it cannot "jump" to values $2 \cdot \pi \cdot k$ for $k \neq 0$, hence it is always equal to 0 . So, the above polynomial is identically 0 , hence all its coefficients $t_{5 i_{2} \ldots i_{p}}^{j_{1} \ldots j_{q}}$ are identically 0 .

Similarly, we can prove that $t_{5}^{i_{1} \ldots}=0$ if $i_{1} \neq 5$, so $t_{5}^{5 \ldots 5}$ is indeed the only non-zero component of $t \cdots$ for which one of the lower indices is 5 . For this component, the fact that $h \cdot t_{5}^{5 \ldots 5} \cdot p_{5} \cdot \ldots \cdot p_{5}=2 \cdot \pi \cdot k$, where $p_{5}=1 / h$, leads to the desired formula for $t_{5}^{5 \ldots 5}$.

To complete the proof, let us assume that the two sets of coefficients $t \cdots$ and $s \ldots$ define the same $K$-tensor. This means that for their difference $\delta \ldots$, the sum

$$
\sum_{i_{1}, \ldots, i_{p}, j_{1}, \ldots, j_{q}} \delta_{i_{1} \ldots i_{p}}^{j_{1} \ldots j_{q}} \cdot x^{i_{1}} \cdot \ldots \cdot y^{i_{p}} \cdot z_{j_{1}} \cdot \ldots \cdot u_{j_{q}}
$$

is an integer multiple of $2 \cdot \pi$ for all $x^{i_{1}}, \ldots, y^{i_{p}} \in K$ and $z_{j_{1}}, \ldots, u_{j_{q}} \in K^{*}$. If $p>0$, and one of the indices $j_{1}, \ldots, j_{q}$ is different from 5 , then, as above, we can conclude that the sum is always 0 ,

So, all the corresponding coefficients $\delta^{\cdots}$ are identically 0 . The only possibly non-zero coefficient is $\delta^{5 \ldots 5}$. For this coefficient, the value $\delta^{5 \ldots 5} p_{5} \cdot \ldots \cdot p_{5}$, with $p_{5}=1 / h$, must be proportional to $2 \cdot \pi$ - so $\delta^{5 \ldots 5} \cdot(1 / h)^{p}=2 \cdot \pi \cdot k$ for some integer $k$. Hence, the difference between $s^{5 \ldots 5}$ and $s^{5 \ldots 5}$ is indeed proportional to $2 \cdot \pi \cdot h^{p}$. The proposition is proven.

\section{B. Proof of Proposition 2}

According to Proposition 1, the only possibly non-zero component of a $K$-tensor with 5 as one of the lower indices is the component $t_{5}^{5 \ldots 5}$. All the values $\partial t_{i_{1} \ldots i_{p}}^{j_{1} \ldots j_{q}} / \partial x^{5}$ contain 5 as one of the lower indices, so the only component for which this value can be different from 0 is the one with $p=0$ and $i_{1}=$ $\ldots=i_{p}=5$. For this component, $\partial t^{5 \ldots 5} / \partial x^{5}=2 \cdot \pi \cdot h^{p-1} \cdot k$. Since the $K$-tensor field is continuously differentiable, this value cannot jump to a different value of $k$, so this derivative is constant. Integrating over $x^{5}$, we get the desired formula for the the dependence of this component on $x^{5}$ - as a linear function of $x^{5}$.

\section{Proof of Proposition 3}

Since $K$ locally coincides with $R^{5}$, its continuous automorphisms locally coincide with continuous automorphisms $R^{5} \rightarrow R^{5}$, i.e., with linear transformations

$$
x_{\text {new }}^{5}=A_{5} \cdot x^{5}+\sum_{i=1}^{4} A_{i} \cdot x^{i} ; \quad x_{\text {new }}^{i}=B^{i} \cdot x^{5}+\sum_{j=1}^{4} b_{j}^{i} x^{j} .
$$

If $y^{5}=x^{5}+h$ and $y^{i}=x^{i}$ for all other $i$, then $x$ and $y$ define the exact same point in $K$. Therefore, the new values $x_{\text {new }}$ and $y_{\text {new }}$ must also define the same point, hence $y_{\text {new }}^{i}=x_{\text {new }}^{i}$ for $i=1, \ldots, 4$ (hence $B^{i}=0$ ) and $y_{\text {new }}^{5}-x_{\text {new }}^{5}=$ integer multiple of $h$ (hence $A_{5}$ is an integer).

Reversibility implies that $A_{5}^{-1}$ should also be an integer, hence $A_{5}= \pm 1$.

\section{Proof of Proposition 4}

The condition that the tangent transformation is $K$-linear means that $\partial s^{5} / \partial x^{5}= \pm 1$ (and due to continuity this does not depend on the point $x$, i.e., either it is everywhere equal to 1 , or it is everywhere equal to -1 ), and $\partial s^{i} / \partial x^{5}=0$ for $i<4$. Hence, $s^{5}= \pm x_{5}+f\left(x^{1}, \ldots, x^{4}\right)$ and $s^{i}=f^{i}\left(x^{1}, \ldots, x^{4}\right)$ for $i<5$.

\section{E. Proof of a the Statement About Hertz Potentials}

As we have mentioned, it is possible that $\partial g^{55} / \partial x^{5} \neq 0$. However, if the representation (2) was possible, then we would have

$$
\frac{\partial g^{55}}{\partial x^{5}}=\frac{\partial^{2} \Pi^{555}}{\left(\partial x^{5}\right)^{2}}+\sum_{i=1}^{4} \frac{\partial^{2} \Pi^{55 i}}{\partial x^{i} \partial x^{5}} .
$$

However, according to our general result about components of $K$-tensors, all the terms in the right-hand side are 0 s, so their sum cannot be equal to a non-zero value $\partial g^{55} / \partial x^{5}$. 